\title{
Synthesis of Molecularly Imprinted Polymer for Sterol Separation
}

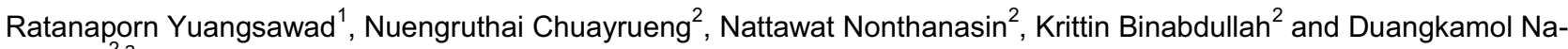 \\ Ranong ${ }^{2, a}$ \\ ${ }^{1}$ Division of Chemical Engineering, Faculty of Engineering, Rajamangala University of Technology Krungthep, 2 Nanglinchii, Sathorn, \\ Bangkok 10120, Thailand \\ ${ }^{2}$ Department of Chemical Engineering, Faculty of Engineering, King Mongkut's Institute of Technology Ladkrabang, 1 Chalongkrung 1 , \\ Ladkrabang, Bangkok 10520, Thailand
}

\begin{abstract}
Molecular imprinted polymer (MIP) was prepared by bulk polymerization in acetone using acrylamide as a functional monomer, ethylene glycol dimethacrylate as a crosslinker, stigmasterol as a template and benzoyl peroxide as an initiator. The obtained MIPs were characterized using a scanning electron microscope and a fourier transform infrared spectrophotometer. Performance in sterol adsorption of MIPs prepared under various conditions was investigated using a model solution of phytosterols in heptane, comparing with a nonimprinted polymer (NIP). Statistical analysis revealed that the amounts of crosslinker and template strongly affected the performance of MIP while the amount of solvent slightly affected the performance of MIP. MIP synthesized under the optimal condition had adsorption capacity of $1.28 \mathrm{mgsterols} /$ gads which were 1.13 times of NIP.
\end{abstract}

\section{Introduction}

Due to high energy consumption and depletion of fossil fuel, demand of biodiesel has been increased for several decades. Palm oil and palm fatty acid distillate (PFAD) are mainly used as raw materials for biodiesel production in many countries, especially in South East Asia [1-4]. However, biodiesel producers are suffering from high cost of production. In order to increase the competency of biodiesel in the market, it is necessary to increase economical value of the overall process including oil refinery and biodiesel production.

PFAD is a by-product from palm oil refinery. It consists of free fatty acids $(81.7 \%)$, glycerides $(14.4 \%)$ and several high valuable bioactive compounds such as vitamin E, phytosterols and squalene [5]. Phytosterols have several biological activities such as cholesterol lowering effect, anti-inflammatory, anti-atherogenicity, anti-cancer and anti-oxidation. Therefore, applications of phytosterols in pharmaceutical, functional food and cosmetics industries are promising [6].

Recovery of phytosterols from PFAD with reasonable cost should increase economical value of overall process of palm oil refinery and biodiesel production. Several processes including chemical transformation and physical separation steps have been developed mainly to recover phytosterols from soybean oil fatty acid distillate and tall oil [6-9]. These processes usually include the step with high energy consumption such as molecular distillation under high vacuum condition, crystallization at low temperature, recovery of solvent from the solvent extraction and supercritical fluid extraction. Therefore, it is necessary to develop the more environmental friendly and energy saving process for phytosterols recovery.

Adsorption has been widely applied in an industrial process to separate minor contaminants from liquid and gas. It has high potential for application of phytosterols recovery. Since PFAD consists of several types of minor components [5], one of the key factors for the success of the separation of phytosterols by adsorption is selectivity of the adsorbent towards phytosterols.

Molecularly imprinted polymers (MIPs) have been applied in various fields requiring high selectivity to particular molecules such as biosensor development, chromatography and catalysis [10-12]. In this work, MIPs were synthesized from acrylamide (monomer), ethylene glycol dimethacrylate (crosslinker) and stigmasterol (template) by bulk polymerization under various conditions. Amounts of crosslinker, template and solvent were selected as synthesis parameters. Properties of the obtained MIPs were observed using SEM and FT-IR techniques. Batch adsorption was performed to evaluate the performance of the obtained MIPs in sterol adsorption using a model solution of phytosterols in n-heptane. Performance of MIPs was compared with a nonimprinted polymer (NIP).

\section{Experimental}

\subsection{Synthesis of molecularly imprinted polymers}

Acrylamide (monomer), ethylene glycol dimethacrylate (EGDMA, crosslinker), stigmasterol (template), benzoyl

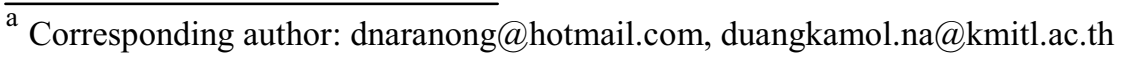


peroxide (initiator) and acetone (solvent) were mixed in a $60 \mathrm{ml}$ glass bottle. In order to remove oxygen, nitrogen gas was purged through the solution for $10 \mathrm{~min}$. Then the solution was prepolymerized at $60{ }^{\circ} \mathrm{C}$ for $1 \mathrm{~h}$ by shaking in an orbital shaker at $220 \mathrm{rpm}$. After prepolymerization, the temperature in the shaker was raised to $60{ }^{\circ} \mathrm{C}$ and the polymerization was allowed to proceed at this condition for $24 \mathrm{~h}$. The obtained solid was washed several times with distilled water to remove all unreacted reagents. To ensure that all the unreacted reagents were completely removed from the obtained solid, water was separated from the solid by centrifugation at $4,000 \mathrm{rpm}$ for $30 \mathrm{~min}$ and its absorbance was measured using UVspectrophotometer. To remove the stigmasterol template from the obtained solid, Soxhlet extraction was performed at $120{ }^{\circ} \mathrm{C}$ for $24 \mathrm{~h}$ using solution consisting of acetonitrile ( $85 \mathrm{vol} \%)$, water $(9.9 \mathrm{vol} \%)$, methanol (5 vol $\%)$ and acetic acid $(0.1 \mathrm{vol} \%)$ as extracting solvent. To ensure that the template was completely removed, the obtained MIPs mixed with n-heptane and stirred at $30{ }^{\circ} \mathrm{C}$ for $3 \mathrm{~h}$ and the solution was taken for analysis of stigmasterol using a gas chromatograph with flame ionized detector (GC-2010plus; Shimadzu).

Different MIPs were prepared by varying ratios of EGDMA, stigmasterol and acetone to acrylamide in the ranges of $2: 1$ to $4: 1$ (mol:mol), $0.1: 1$ to $0.3: 1$ (mol:mol) and $4: 1$ to $8: 1$ (mol:mol), respectively. Amount of benzoyl peroxide was fixed at 0.32 mole per mole of acrylamide. Conditions for the MIPs synthesis were determined using a MINITAB program according to Central Composite Design (CCD) method. The result is summarized in Table 1.

Table 1. Condition for MIP synthesis determined by CCD method. (1 mmol of monomer, initiator $0.32 \mathrm{mmol}$ )

\begin{tabular}{|c|c|c|c|}
\hline No. & $\begin{array}{c}\text { crosslink } \\
(\mathbf{m m o l})\end{array}$ & $\begin{array}{c}\text { template } \\
(\mathbf{m m o l})\end{array}$ & $\begin{array}{c}\text { solvent } \\
(\mathbf{m l})\end{array}$ \\
\hline 1 & 2.5 & 0.15 & 5 \\
\hline 2 & 3.5 & 0.15 & 5 \\
\hline 3 & 2.5 & 0.25 & 5 \\
\hline 4 & 3.5 & 0.25 & 5 \\
\hline 5 & 2.5 & 0.15 & 7 \\
\hline 6 & 3.5 & 0.15 & 7 \\
\hline 7 & 2.5 & 0.25 & 7 \\
\hline 8 & 3.5 & 0.25 & 7 \\
\hline 9 & 2 & 0.2 & 6 \\
\hline 10 & 4 & 0.2 & 6 \\
\hline 11 & 3 & 0.1 & 6 \\
\hline 12 & 3 & 0.3 & 6 \\
\hline 13 & 3 & 0.2 & 4 \\
\hline 14 & 3 & 0.2 & 8 \\
\hline Std & 3 & 0.2 & 6 \\
\hline
\end{tabular}

For further discussion, the MIP obtained at the condition of monomer: crosslinker : template : solvent $=$ $1: 3: 0.2: 6$ was named as "MIP-STD". The solid sample obtained under this condition before extraction of template was named as "T-MIP-STD". Nonimprinted polymer (NIP) was prepared using this ratio by the same procedure without addition of stigmasterol template.

\subsection{Characterization of molecularly imprinted polymers}

SEM images were taken to observe morphology of the samples using EVO®MA10 (ZEISS). Extra high tension voltage level was $15.00 \mathrm{kV}$. Fourier transform infrared spectra were measured using IRPrestige-21 (Shimadzu, Japan) equipped with MIRacle ATR (PIKE Technologies, Inc.) with a resolution of $4 \mathrm{~cm}^{-1}$.

\subsection{Adsorption test}

Model solution was prepared from sterol mixed, containing campesterol (23.6 wt \%), stigmasterol (28.2 $w t \%)$ and $\beta$-sitosterol (48.2 wt \%), and n-heptane. Concentration of total sterols in the model solution was set at $140 \mu \mathrm{g} \cdot \mathrm{g}^{-1}$. Batch adsorption was performed as follows. $20 \mathrm{ml}$ of the prepared model solution was mixed with $1 \mathrm{wt} \%$ of MIP or NIP and then the mixture was shaken at $220 \mathrm{rpm}, 30^{\circ} \mathrm{C}$ for $6 \mathrm{~h}$.

Samples (c.a. $0.1 \mathrm{~g}$ ) before and after adsorption were taken for quantitative analysis of campe-, stigma- and $\beta$ sitosterol. Analysis was performed using a gas chromatograph connected with flame ionized detector (GC-2010plus; Shimadzu). Peak separation was achieved using a ZB-5HT capillary column (30 m in length, 0.32 $\mathrm{mm}$ in internal diameter, $0.1 \mu \mathrm{m}$ in film thickness; Phenomenex). Tricaprin was used as an internal standard. Calibration curve of sterols in n-heptane was made in the range of $0-120 \mu \mathrm{g}$. The obtained calibration curve was expressed as $W_{\text {sterol }}=13.075\left(A_{\text {sterol }} / A_{\text {Tricaprin }}\right)+0.6042$ with $R^{2}=0.9997$.

Performance of the adsorbent was evaluated from percentage of sterol adsorption (\%Ads: \%, Eq. (1)), adsorption capacity $\left(q_{\mathrm{i}}: \mathrm{g}_{\text {sterols }} / \mathrm{g}_{\mathrm{ads}}\right.$, Eq. (2)) and selectivity based on stigmasterol $\left(S_{\mathrm{i}}: \%\right.$, Eq. (3)).

$$
\begin{gathered}
\% \mathrm{Ads}=\frac{C_{0}-C}{C_{0}} \times 100 \\
q=\frac{\left(C_{0}-C\right) W_{\text {solution }}}{W_{\text {ads }}} \\
S_{\mathrm{i}}=\left(\frac{W_{\mathrm{i}, 0}-W_{\mathrm{i}}}{W_{\mathrm{i}, 0}}\right)\left(\frac{W_{\text {stigma }, 0}}{W_{\text {stigma }, 0}-W_{\text {stigma }}}\right)
\end{gathered}
$$

\subsection{Statistical analysis}

ANOVA analysis was performed in order to investigate effects of the synthesis parameters on the adsorption performance of the obtained MIP and to predict the optimum condition of MIP synthesis.

\section{Results and Discussion}




\subsection{Polymer characterization}

All the synthesized MIPs and NIP were white fine powder and were not dissolved in water and n-heptane. Figures 1(a) and 1(b) show SEM images of MIP-STD with the magnifications of 500 and 20,000, respectively. SEM images reveal that most of the particles had the size smaller than $100 \mu \mathrm{m}$ and the surface of MIP-STD was not uniformed. Pores with the diameter less than $1 \mu \mathrm{m}$ and cavities with the size in the order of $2 \mu \mathrm{m}$ or larger are observed. SEM images of MIP synthesized under other conditions (not shown) had characteristic similar to those of MIP-STP. Figures 1(c) and 1(d) show SEM images of NIP. No large cavity is observed. Therefore, the large cavities in MIP-STP should be considered as a result of existing of stigmasterol during the polymerization and they were formed after removal of the template.

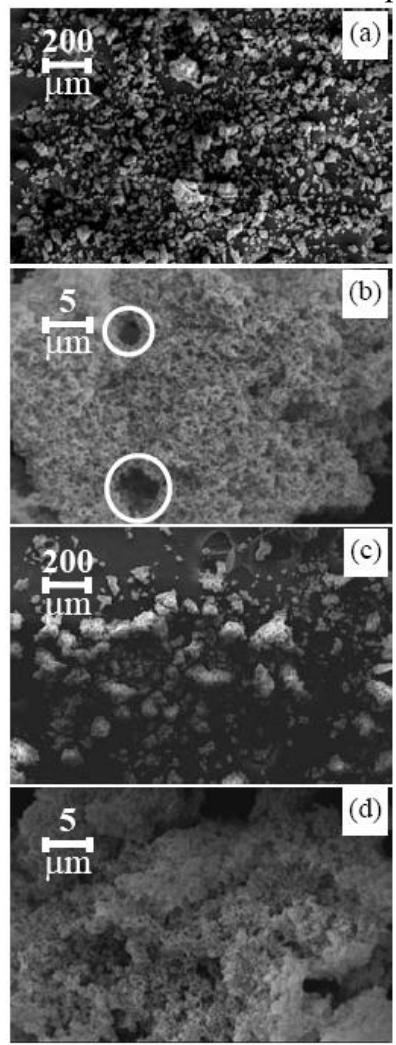

Figure 1. Typical SEM images of MIP-STD (a, b), NIP (c, d).

Figure 2 shows FT-IR spectra of NIP and T-MIP-STD. Identification of absorbed peaks was performed by using database of FT-IR equipment. Characteristics of both spectra were similar. Both spectra had small broad peaks of N-H vibration around $3460 \mathrm{~cm}^{-1}$, small sharp peak of $\mathrm{C}-\mathrm{H}$ vibration at $2960 \mathrm{~cm}^{-1}$, large sharp peak of $\mathrm{C}=\mathrm{O}$ vibration at $1730 \mathrm{~cm}^{-1}$ and peak of $\mathrm{C}-\mathrm{N}$ vibration at 1400 $\mathrm{cm}^{-1}$. These peaks were identified as the N-H, C=O, C-N and $\mathrm{C}-\mathrm{H}$ in the structure of acrylamide-EGDMA polymer. Since no absorption band of $\mathrm{C}=\mathrm{C}$ (at $1600 \mathrm{~cm}^{-1}$ ) was observed in these spectrum and both acrylamide monomer and EGDMA have $\mathrm{C}=\mathrm{C}$ in their structures, this result indicates that unreacted acrylamide and EGDMA were completely removed from the obtained NIP-STD and T-MIP-STD by water washing. Comparison of the spectra of NIP and T-MIP-STD reveals that NIP and TMIP-STD had almost the same chemical structures.
However, the relative intensity of $\mathrm{C}-\mathrm{H}$ peak to other peaks in the spectrum of NIP was less than the relative intensity observed in the spectrum of T-MIP-STD. This result indicated existing of stigmasterol, containing $\mathrm{C}-\mathrm{H}$ bond, in T-MIP-STD.

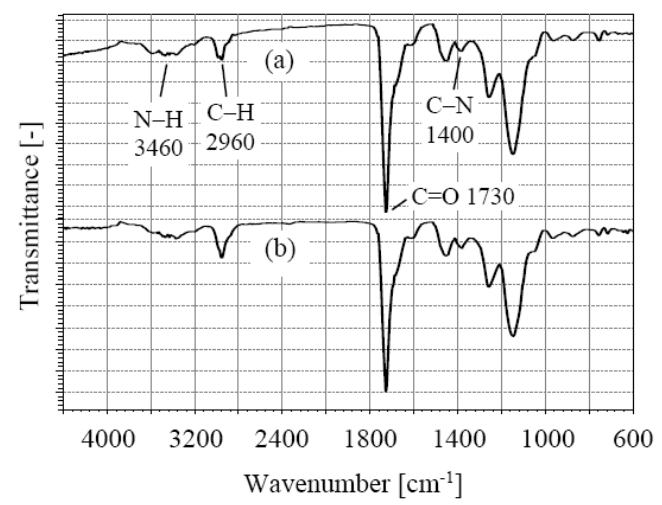

Figure 2. FT-IR spectra of (a) NIP-STD and (b) T-MIP-STD.

\subsection{Performance of phytosterols adsorption}

Figure 3 shows the performance in sterol adsorption of MIPs comparing with NIP. As shown in the figure, MIPs prepared at the condition number of 2, 4, 14 and STD adsorbed larger amount of sterols comparing with NIP. Moreover, adsorption experiment revealed that all the prepared MIPs adsorbed campe-, stigma- and $\beta$-sitosterol with almost the same percentage of the original amounts presenting in the model solution; $S_{\text {campesterol }} \approx S_{\beta \text {-sitosterol }} \approx 1$. No specific selectivity towards stigmasterol adsorption should be a result of very similar molecular structures of these sterols. This result should be considered as the possibility of applying MIP in recovery of all types of sterol from fatty acid distillates. Therefore, adsorption capacity was calculated from total amount of campesterol, stigmasterol and $\beta$-sitosterol adsorbed on the adsorbents.

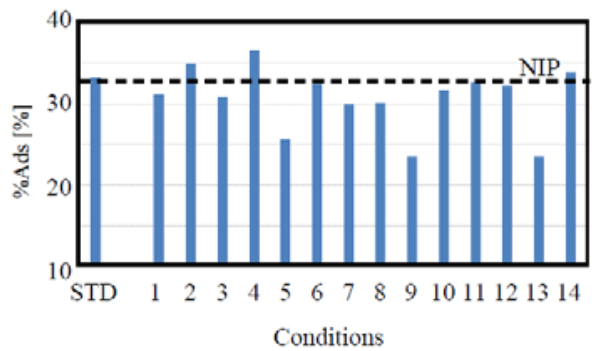

Figure 3. Performance of molecularly imprinted polymers in sterol adsorption at $30^{\circ} \mathrm{C}, 6 \mathrm{~h}$; (- - ) NIP.

\subsection{Effect of synthesis parameters on adsorption performance of MIP}

The relation between percentage of sterol adsorption and amounts of crosslinker, template and solvent obtained from statistical analysis are shown in Figure 4(a) - 4(c).

As shown in Figure 4(a), in the investigated range, increasing the amount of crosslink improved the performance in sterol adsorption of the obtained MIPs. The effect was more significant when the amount of template was low. However, increasing the amount of 
template $(0.1$ to $0.3 \mathrm{mmol})$ non-monotonously affected adsorption performance of the obtained MIPs. The lowest efficiency was observed from the MIP synthesized at the template $(0.1$ to $0.3 \mathrm{mmol})$ non-monotonously affected adsorption performance of the obtained MIPs. The lowest efficiency was observed from the MIP synthesized at the template $(0.1$ to $0.3 \mathrm{mmol})$ non-monotonously affected adsorption performance of the obtained MIPs. The lowest efficiency was observed from the MIP synthesized at the amount of template around $0.15 \mathrm{mmol}$. Figure 4(b) shows that increase of the amount of crosslink improved the performance in sterol adsorption of the obtained MIPs whereas the amount of solvent insignificantly affected the performance in sterol adsorption of the obtained MIPs. Effect of the amount of template on the performance in sterol adsorption of the obtained MIPs shown in Figure 4(c) agrees well with the same effect shown in Figure 4(a).
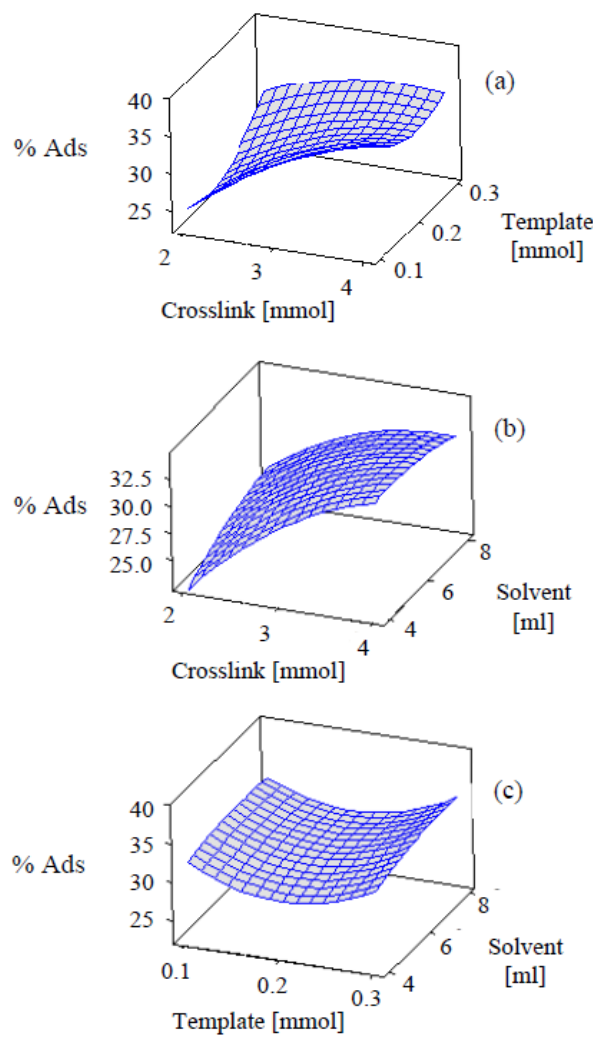

Figure 4. Effects of synthesis parameters on performance of MIPs. Synthesis conditions: (a) volume of solvent $=5 \mathrm{ml}$, (b) amount of template $=0.20 \mathrm{mmol}$, amount of crosslink $=3 \mathrm{mmol}$.

According to response analysis, the optimal amounts of crosslinker, template and solvent were $3.5 \mathrm{mmol}, 0.3$ mmol and $7 \mathrm{ml}$, respectively and the MIP synthesized under this optimized condition had adsorption capacity of $1.28 \mathrm{mg}_{\text {sterols }} / \mathrm{g}_{\text {ads }}$ or 1.13 times of NIP.

\section{Conclusions}

Molecularly imprinted polymer (MIP) using for sterol separation was successfully synthesized by bulk polymerization of acrylamide and EGDMA using stigmasterol as template, acetone as solvent and benzoyl peroxide as initiator. MIP had large cavity formed from stigmasterol in its structure. Performance of MIP in sterol adsorption was significantly affected by the amounts of crosslinker and template but slightly affected by the amount of solvent used in the preparation of MIP. MIP synthesized at the optimal condition had adsorption efficiency of 1.13 times of NIP.

\section{Acknowledgement}

The authors would like to acknowledge financial support from National Research Council of Thailand.

\section{Nomenclature}

$\%$ Ads percentage of sterol adsorption

$C$ total concentration of campesterol, stigmasterol and $\beta$-sitosterol in the model solution at $6 \mathrm{~h}$

$C_{0} \quad$ total concentration of campesterol, stigmasterol and $\beta$-sitosterol in the model solution at $0 \mathrm{~h}$

$C_{\mathrm{i}} \quad$ concentration of species "i" in the model solution

$q \quad$ capacity of sterols adsorption

$W_{\mathrm{i}} \quad$ weight of species "i" in the model solution at $6 \mathrm{~h}$

$W_{\mathrm{i}, 0} \quad$ weight of species " $\mathrm{i}$ " in the model solution at $0 \mathrm{~h}$

$W_{\text {sol }}$ weight of model solution

\section{Subscript}

i campesterol, stigmasterol or $\beta$-sitosterol

\section{References}

1. E.N. Ali, C.I. Tay, Procedia Engineering, 53, 7 (2013)

2. V.V. Kuss, A.V. Kuss, R. G. Rosa, D.A.G. Aranda, Y.R.Cruz, Renew. Sust. Energ. Rev., 50, 1013 (2015)

3. M.H. Mosarof, M.A.Kalam, H.H. Masjuki, A.M. Ashraful, M.M. Rashed, H.K. Imdadul, I.M. Monirul, Energ. Convers. Manage., 105, 617 (2015)

4. S. Mekhilef, S. Siga, R. Saidur, Renew. Sust. Energ. Rev., 15, 1937 (2011)

5. A.G.M. Top, Lipid Technol., 22, 11 (2010)

6. P. Fernandes, J.M.S. Cabral, Bioresour. Technol., 98, 2335 (2007)

7. M.-J. Dumont, S. S. Narine, Food Res. Int., 40, 957 (2007)

8. H. Yang, F. Yan, D. Wu, M. Huo, J. Li, Y. Cao, Y. Jiang, Bioresour. Technol., 101, 1471 (2010)

9. C. J. Chang, Y.-F. Chang, H. Lee, J. Lin, P.-W. Yang, Ind. Eng. Chem. Res., 39, 4521 (2000)

10. W. J. Cheong,, S. H. Yang, F. Ali, J. Sep. Sci., 36, 609 (2013)

11. N. Verma, A. Bhardwaj, Appl. Biochem. Biotechol., 175, 3093 (2015)

12. W. Li, S. Li, Adv. Polymer Sci., 206, 191 (2007) 